\title{
Efficacy and Safety of a New 20\% Immunoglobulin Preparation for Subcutaneous Administration, IgPro20, in Patients With Primary Immunodeficiency
}

\author{
John B. Hagan • Mary B. Fasano • Sheldon Spector • \\ Richard L. Wasserman • Isaac Melamed • Mikhail A. Rojavin • \\ Othmar Zenker • Jordan S. Orange
}

Received: 15 February 2010 / Accepted: 13 April 2010 /Published online: 8 May 2010

(C) The Author(s) 2010. This article is published with open access at Springerlink.com

\begin{abstract}
Subcutaneous human IgG (SCIG) therapy in primary immunodeficiency (PID) offers sustained IgG levels throughout the dosing cycle and fewer adverse events (AEs) compared to intravenous immunoglobulin (IVIG). A phase I study showed good local tolerability of IgPro20, a new 20\% liquid SCIG stabilized with L-proline.
\end{abstract}

\section{J. B. Hagan $(\square)$}

Division of Allergic Diseases, Mayo Clinic,

200 First St. S.W.,

Rochester, MN 55905, USA

e-mail: hagan.john@mayo.edu

M. B. Fasano

University of Iowa Hospitals \& Clinics,

Iowa City, IA, USA

\section{S. Spector}

California Allergy \& Asthma Medical Group Inc,

Los Angeles, CA, USA

R. L. Wasserman

DallasAllergyImmunology and Medical City Children's Hospital,

Dallas, TX, USA

I. Melamed

IMMUNOe International Research Centers,

Centennial, CO, USA

M. A. Rojavin

CSL Behring, LLC,

King of Prussia, PA, USA

O. Zenker

CSL Behring GmbH,

Marburg, Germany

J. S. Orange

Division of Immunology, Children's Hospital of Philadelphia,

Philadelphia, PA, USA
A prospective, open-label, multicenter, single-arm, phase III study evaluated the efficacy and safety of IgPro20 in patients with PID over 15 months. Forty-nine patients (572 years) previously treated with IVIG received weekly subcutaneous infusions of IgPro20. The mean serum IgG level was $12.5 \mathrm{~g} / \mathrm{L}$. No serious bacterial infections were reported. There were 96 nonserious infections (rate 2.76/ patient per year). The rate of days missed from work/school was 2.06/patient per year, and the rate of hospitalization was $0.2 /$ patient per year. Ninety-nine percent of AEs were mild or moderate. No serious, IgPro20-related AEs were reported. IgPro20 effectively protected patients with PID against infections and maintained serum IgG levels without causing unexpected AEs.

Keywords Subcutaneous immunoglobulin (SCIG) . primary immunodeficiency · local tolerability · serum IgG trough levels $\cdot \mathrm{L}$-proline $\cdot$ home infusion therapy

\section{Introduction}

Common variable immunodeficiency (CVID) and X-linked agammaglobulinemia (XLA) are primary immunodeficiency (PID) disorders that predispose patients to recurrent infections and chronic lung disease [1-6]. Patients require immunoglobulin replacement therapy, which can be administered intravenously (IVIG) or subcutaneously (SCIG) [714]. Subcutaneous administration produces stable serum IgG levels and is associated with fewer systemic adverse events (AEs). Although head-to-head studies have not been performed, retrospective analyses and two crossover trials have demonstrated a lower incidence of systemic AEs in patients receiving SCIG compared to IVIG [15-23]. Recent- 
ly, SCIG has gained recognition due to its suitability for selfinfusion and home therapy, both offering greater flexibility to patients [20].

IgPro20 is a new $20 \%$ liquid SCIG product with high purity ( $\geq 98 \%$ IgG) manufactured from human plasma of more than 15,000 donors by a process identical to that of IgPro10 (Privigen ${ }^{\circledR}$ ), which has an established rigorous pathogen safety profile [24]. The high IgG concentration in IgPro20 allows for infusion of lower volumes compared to currently available $16 \% \operatorname{IgG}$ products administered at equivalent dose. This potentially solves an existing problem with SCIG therapy for some patients, namely, the number of infusion sites or doses required to provide adequate $\operatorname{IgG}$ replacement. Despite high protein concentration, IgPro20 has outstanding solution properties and can be administered at relatively high infusion rates-a substantial technical achievement. Formulation with L-proline and Polysorbate 80 allows storage of IgPro20 for up to 24 months at $25^{\circ} \mathrm{C}$ without any loss of functional activity (Maeder et al., in preparation).

We report results on local tolerability in healthy volunteers from a phase I study, as well as efficacy and tolerability of IgPro20 in patients with PID from a recently completed prospective, open-label, multicenter, single-arm, phase III study (NCT00419341).

\section{Methods}

Phase I Local Tolerability Study in Healthy Subjects

\section{Subjects}

Twenty-eight healthy, male, white subjects aged 18 to 45 years were recruited. Inclusion criteria included a body mass index (BMI) of $21-27 \mathrm{~kg} / \mathrm{m}^{2}$, no clinically significant medical history, and good health (as determined by a detailed medical history, complete physical examination, electrocardiogram, and clinical laboratory screening). Subjects were excluded in case of evidence of any clinically relevant pathology that could interfere with the study results or put the subject's safety at risk.

This study was conducted in accordance with the International Conference on Harmonization (ICH) Good Clinical Practice (GCP) guidelines, and the Declaration of Helsinki (version of 1996). The study protocol and all other study documents were approved by the relevant Independent Ethics Committees. Subjects signed an informed consent prior to entering the study.

\section{Study Design}

This was a single-center, randomized, four-way crossover, assessment-blinded study. The study objective was to compare the local tolerability of IgPro16 and IgPro20 with Vivaglobin ${ }^{\circledR}$.

IgPro16 and IgPro20 are $16 \%$ and $20 \%$ liquid human IgG products, respectively, formulated with $28.8 \mathrm{mg} / \mathrm{mL}$ $(250 \mathrm{mmol} / \mathrm{L})$ of L-proline and $20 \mathrm{mg} / \mathrm{L}$ of Polysorbate 80 at $\mathrm{pH}$ 4.8; Vivaglobin ${ }^{\circledR}$ is a $16 \%$ liquid human $\operatorname{IgG}$ formulated with $22.5 \mathrm{mg} / \mathrm{mL}(300 \mathrm{mmol} / \mathrm{L})$ of glycine at pH 6.4-7.2. Each patient received a single subcutaneous dose of IgPro16 $15 \mathrm{~mL}$, IgPro20 $15 \mathrm{~mL}$, IgPro20 $12 \mathrm{~mL}$, or Vivaglobin $15 \mathrm{~mL}$ at a single abdominal site on day 1 at $25 \mathrm{~mL} / \mathrm{h}$. The next test samples were administered at weekly intervals at different abdominal sites.

The primary end point was assessment of local tolerability from the start of infusion to 72 hours after the end of infusion. Local tolerability included pain (assessed by subjects), erythema, edema/induration, itching, and local heat (assessed by a treatment-blinded investigator).

\section{Tolerability Assessments}

Assessments were performed by treatment-blinded investigators after the end of infusions. Erythema and edema were evaluated using 5-point scales ranging from none (score 0) to severe (score 4). Itching and local heat were evaluated using a 4-point scale ranging from none (score 0 ) to severe (score 3). Pain was evaluated by the subjects using a 100-mm Visual Analogue Scale (VAS) ranging from 0 (no pain) to 100 (unbearable pain).

The results of the assessments of local tolerability parameters were not documented as AEs. Local reactions were recorded as AEs when the symptoms/signs led to infusion stop, required concomitant medication, or had an impact on the general condition of the subject as judged by the investigator.

\section{Phase III Efficacy and Safety Study in Patients With PID}

\section{Patients}

Males or females aged 5 to 72 years diagnosed as having CVID, as defined by the Pan-American Group for Immunodeficiency and European Society for Immunodeficiencies, or XLA, as determined by the investigator, were enrolled in the study. Patients were receiving IVIG therapy at regular 3- or 4-week intervals for at least 3 months prior to study start. Patients switching from a Privigen study in PID (NCT00322556) were required to have had at least three documented serum $\operatorname{IgG}$ trough levels of $\geq 5 \mathrm{~g} / \mathrm{L}$ for the last 3 months prior to enrollment in this study. Patients treated with other IVIG products were required to have had at least one documented serum IgG trough level of $\geq 5 \mathrm{~g} / \mathrm{L}$ during the previous 6 months. 
Exclusion criteria included newly diagnosed PID (previously untreated with IVIG), evidence of an active serious infection, malignancies of lymphoid cells and immunodeficiency with thymoma, known hyperprolinemia, hypoalbuminemia, protein-losing enteropathies, any proteinuria, allergic reactions to immunoglobulins or other blood products, known antibodies to IgA, treatment with steroids (oral or parenteral, $\geq 0.15 \mathrm{mg}$ of prednisone equivalent $/ \mathrm{kg}$ per day) or other systemic immunosuppressants, pregnancy, breast feeding planned pregnancy during the course of the study, and any condition that was likely to interfere with evaluation of the study medication or satisfactory conduct of the study.

This study was conducted in accordance with the ICH GCP guidelines and the Declaration of Helsinki (version of 1996). The study protocol and all other study-related documents were reviewed and approved by the appropriate local institutional review boards. Written informed consent was obtained from all patients or patients' parents or legal guardians.

\section{Study Design}

This was a prospective, open-label, multicenter, single-arm, phase III study with a 12-week wash-in/wash-out period followed by a 12-month efficacy period. Within the main study, a two-part pharmacokinetic substudy was conducted in a subset of patients $(n=19)$. Apart from evaluation of IgPro20 pharmacokinetic parameters, the pharmacokinetic substudy provided data for dose adjustments in the efficacy period of the main study.

The initial weekly dose of IgPro20 administered during the wash-in/wash-out period was the calculated average weekly dose of the last three IVIG infusions received prior to the start of this study, multiplied by 1.30, a dose adjustment coefficient used in a study with Vivaglobin [25]. In the efficacy period, patients not participating in the pharmacokinetic substudy had their IgPro20 doses adjusted by applying a mean dose adjustment coefficient of 1.53 , calculated from individual dose adjustments in pharmacokinetic patients (Wasserman et al., manuscript in preparation).

Premedication taken on the same day prior to infusion to reduce AEs associated with SCIG infusion was not allowed. Oral and parenteral steroids as concomitant medication were allowed if the average daily dose was $<0.15 \mathrm{mg}$ of prednisone equivalent $/ \mathrm{kg}$ per day. Local anesthetics could be used before infusion to reduce the pain associated with needle insertion if required.

IgPro20 was administered subcutaneously using Cane Crono PCA-50 infusion pumps (Cane S.R.L., Turin, Italy). Potential injection sites were located on the abdomen, thighs, upper arms, and/or lateral hip, with actual injection points changed every week. Up to four injection sites were used simultaneously with a maximum of two pumps, each with bifurcated tubing feeding two catheters. The initial volume per injection site was $15 \mathrm{~mL}$ and could be increased to a maximum of $25 \mathrm{~mL}$, depending on tolerability. The infusion rate was to be $\leq 15 \mathrm{~mL} / \mathrm{h}$ per site for the first infusion and could subsequently be increased stepwise up to $25 \mathrm{~mL} / \mathrm{h}$ per site depending on tolerability.

The first three infusions were supervised and served as training for the patients or their parents/guardians. Patients administered further infusions themselves, with supervised infusions during each study site visit (first four infusions and every fourth infusion thereafter).

\section{Efficacy and Safety Assessments}

The primary efficacy end point was the annual rate of serious bacterial infections (SBIs) per patient in the modified intention-to-treat (MITT) population comprising all subjects treated with study medication during the efficacy period (starting with Week 13). Target rate of SBIs was 1.0 SBI per patient per year, as recommended by the Food and Drug Administration (FDA) [26]. SBIs were defined as bacterial pneumonia, bacteremia/septicemia, osteomyelitis/septic arthritis, bacterial meningitis, and visceral abscess. Each AE identified as a potential SBI was retrospectively adjudicated by a review committee to determine whether the event fulfilled the predefined criteria for SBIs.

Secondary efficacy end points included rate of SBIs in the ITT population, number of infection episodes, number of days missed from work/school/day care or inability to perform normal activities due to infections (abbreviated further as number of days missed from work/school), number of days of hospitalization due to infections, use of antibiotics for infection prophylaxis or treatment, and trough serum IgG levels. Information about secondary efficacy end points was collected via patient diaries. Trough serum IgG levels were measured before every infusion administered at the study site (every 4 weeks).

Information on AEs, including local reactions, was collected continuously by patients (diary) and investigators (at visits).

For the analysis of local reactions, the category of local reactions was created as derived from the MedDRA category "General disorders and administration site conditions" and combined the following MedDRA preferred terms: injection site reaction, injection site bruising, infusion site scab, injection site cyst, injection site eczema, injection site irritation, injection site nodule, and injection site pain. Local reactions were assessed by both patients and investigators. Patients assessed the overall perception of local reactions at $24 \pm 3$ hours after the end of infusion via diaries, using a 5 -point scale $(0=$ none, very slight, slight, moderate, severe). Appropriate completion of the 
diary was monitored at every visit to the study site. Investigators evaluated local reactions (erythema, edema/ induration and itching, local pain, and local heat) independently at 15-45 minutes postinfusion for the first four infusions at the study site and at every visit to the study site thereafter, using symptom-specific 5-point scales, except for edema, which was measured using two diameters in $\mathrm{mm}$.

Vital signs, including blood pressure, heart rate, and body temperature, were evaluated at screening and before and after each infusion administered at the study site (first four infusions and every fourth infusion thereafter). Clinical laboratory parameters were determined at screening; before infusions $1,4,28$, and 40 ; and at the completion visit.

A direct Coombs' test was performed before and shortly after infusions 1 and 4, on day 3 of week 28, before and after infusion 64, 2 or 3 days after infusion 64 , and at the completion visit. If a positive direct Coombs' test result was accompanied by a decrease of $\geq 2.0 \mathrm{~g} / \mathrm{dL}$ in hemoglobin compared to the preinfusion 64 , then the test results were followed-up by extensive additional testing. A decrease of $\geq 2.0 \mathrm{~g} / \mathrm{dL}$ in hemoglobin together with an increase in lactate dehydrogenase and a decrease in serum haptoglobin were considered indicative of a potential intravascular hemolysis.

\section{Statistical Methodology}

A sample size of 32 patients was calculated to provide $80 \%$ power of detecting SBIs, provided the true SBI rate was
0.5. To allow for a moderate to high discontinuation rate, a sample size of 50 patients was planned.

Efficacy analyses were carried out on the MITT population. Safety analyses were carried out in the ITT population, defined as all subjects treated with the study medication during the entire study period.

Efficacy analyses were restricted to the efficacy period (week 13-to completion visit). The annual rate of SBIs per patient was estimated along with its upper one-sided 99\% confidence interval (CI). The number of infection episodes was estimated with two-sided $95 \%$ CIs. Variables concerning demographic and background characteristics and secondary efficacy variables were summarized descriptively. No imputation of missing data was made.

\section{Results}

Phase I Local Tolerability Study in Healthy Subjects

All 28 subjects randomized completed the study.

The mean $( \pm \mathrm{SD})$ age of subjects was $34( \pm 5.97)$ years (range $22-45$ years). The mean $( \pm \mathrm{SD})$ body weight (bw) was $75.5( \pm 7.4) \mathrm{kg}$, with a mean $( \pm \mathrm{SD}) \mathrm{BMI}$ of $23.4 \mathrm{~kg} / \mathrm{m}^{2}$ $\left( \pm 1.58 \mathrm{~kg} / \mathrm{m}^{2}\right)$.

The subjective assessment of pain associated with infusion based on the $100 \mathrm{~mm}$ VAS scale was very low (Table 1). Both maximum pain and mean pain were lower with IgPro20 compared to Vivaglobin. Treatment differences for mean pain were statistically significant for IgPro16 and IgPro20 (12 mL) versus Vivaglobin ( $p=$ 0.0328 and $p=0.0205$, respectively).

Table 1 Local Tolerability: Pain (Healthy Subjects)

\begin{tabular}{|c|c|c|c|c|}
\hline Variable & IgPro16 (15mL) & IgPro20 $(15 \mathrm{~mL})$ & IgPro20 $(12 \mathrm{~mL})$ & Vivaglobin $(15 \mathrm{~mL})$ \\
\hline Subjects treated ${ }^{\mathrm{a}}$ & $28(100 \%)$ & $28(100 \%)$ & $28(100 \%)$ & $28(100 \%)$ \\
\hline Maximum pain ${ }^{\mathrm{b}}(\mathrm{mm})$, mean $(\mathrm{SD})$ & $6.8(12.52)$ & $7.6(12.46)$ & $6.9(12.13)$ & $9.3(14.18)$ \\
\hline Mean pain ${ }^{\mathrm{b}}(\mathrm{mm})$, mean (SD) & $2.36(5.029)$ & $2.84(3.612)$ & $2.24(4.240)$ & $3.78(7.244)$ \\
\hline Variable & \multicolumn{2}{|l|}{ Comparison } & Difference $^{c}$ & Two-sided 90\% CI $(p)$ \\
\hline \multirow[t]{3}{*}{ Maximum pain ${ }^{\mathrm{b}}(\mathrm{mm})$} & \multicolumn{2}{|c|}{ IgPro16 (15 mL)-Vivaglobin } & -2.54 & -4.82 to $-0.26(0.0679)$ \\
\hline & \multicolumn{2}{|c|}{ IgPro20 (15 mL)-Vivaglobin } & -1.64 & -3.92 to $0.64(0.2340)$ \\
\hline & \multicolumn{2}{|c|}{ IgPro20 (12 mL)-Vivaglobin } & -2.43 & -4.71 to $-0.15(0.0801)$ \\
\hline \multirow[t]{3}{*}{ Mean pain ${ }^{\mathrm{b}}(\mathrm{mm})$} & \multicolumn{2}{|c|}{ IgPro16 (15 mL)-Vivaglobin } & -1.41 & -2.50 to $-0.33(0.0328)$ \\
\hline & \multicolumn{2}{|c|}{ IgPro20 (15 mL)-Vivaglobin } & -0.94 & -2.02 to $0.15(0.1545)$ \\
\hline & \multicolumn{2}{|c|}{ IgPro20 (12 mL)-Vivaglobin } & -1.54 & -2.62 to $-0.46(0.0205)$ \\
\hline
\end{tabular}

\footnotetext{
${ }^{\mathrm{a}}$ Results represent number of subjects (\%).

${ }^{\mathrm{b}}$ Pain was assessed by subjects on a 100 -mm VAS; numbers given are from that scale.

${ }^{\mathrm{c}}$ A negative difference indicates a generally higher value of maximum/mean pain under Vivaglobin.
} 
Most of the observed erythema events were "welldefined" or "very slight" (Table 2). Overall, the intensity of erythema was less severe with IgPro20 than with Vivaglobin. While a third of the events with IgPro20 were "very slight" and less than 57\% were "well-defined," 75\% of the events observed with Vivaglobin were "welldefined." At the end of the scheduled observation period on day 4, no erythema was observed in $94(84 \%)$ out of 112 evaluations.

Almost all subjects experienced severe edema/ induration (Table 2), as expected in a subcutaneous infusion. On day 2, 24 hours after the end of infusion, no edema/induration was observed in $31(28 \%)$ of 112 evaluations. At the end of the scheduled observation period on day 4 , no edema/induration was observed in $79(71 \%)$ of 112 evaluations.

Itching was predominantly mild (Table 2). In only 15 of 112 evaluations (13\%), subjects reported mild local heat, with no substantial difference between IgPro16, IgPro20 (12 mL), and Vivaglobin $(15 \mathrm{~mL})$. Local heat started to occur during the infusion and was resolved shortly after the end of infusion. Eight hours after the end of infusion, all reported cases of local heat were resolved.
None of the observed local tolerability events had the characteristics of predefined AEs.

Thus, in a phase I trial, IgPro20 was not inferior in its safety and tolerability when compared to Vivaglobin.

Phase III Efficacy and Safety Study in Patients with PID

\section{Patients}

This phase III study in patients with PID was conducted at 12 sites in the United States. Fifty-two patients were screened, 49 enrolled, 38 completed the wash-in/wash-out period (11 discontinued), and 28 completed the efficacy period (10 discontinued).

The reasons for discontinuation of patients during the wash-in/wash-out period were withdrawal of consent $(n=$ $8)$, AEs $(n=2)$, and disqualifying laboratory results $(n=1)$; the reasons for discontinuation of patients during the efficacy period were withdrawal of consent $(n=6)$, termination of study site $(n=1)$, multiple violations of protocol $(n=1)$, lost to follow-up $(n=1)$, and noncompliance $(n=1)$. Patients who withdrew consent gave the following individual motives for their decision: study time commitment (including weekly site visits in the beginning and/or a
Table 2 Local Tolerability: Incidence of Local Reactions (Healthy Subjects)
For each subject only the highest severity per period is taken into account.

${ }^{a}$ Results represent number of subjects (\%).

\begin{tabular}{lllll}
\hline & IgPro16 $(15 \mathrm{~mL})$ & IgPro20 $(15 \mathrm{~mL})$ & IgPro20 $(12 \mathrm{~mL})$ & Vivaglobin $(15 \mathrm{~mL})$ \\
\hline Subjects treated $^{\mathrm{a}}$ & $28(100)$ & $28(100)$ & $28(100)$ & $28(100)$ \\
Erythema & $23(82.1)$ & $26(92.9)$ & $27(96.4)$ & $28(100)$ \\
None & $5(17.9)$ & $2(7.1)$ & $1(3.6)$ & - \\
Very slight & $9(32.1)$ & $9(32.1)$ & $11(39.3)$ & $4(14.3)$ \\
Well-defined & $12(42.9)$ & $14(50.0)$ & $16(57.1)$ & $21(75.0)$ \\
Moderate to severe & $2(7.1)$ & $2(7.1)$ & - & $3(10.7)$ \\
Severe & - & $1(3.6)$ & - & - \\
Edema/Induration & $28(100)$ & $28(100)$ & $28(100)$ & $28(100)$ \\
None & - & - & - & - \\
Very slight & - & - & - & - \\
Slight & - & - & - & - \\
Moderate & $1(3.6)$ & - & - & - \\
Severe & $27(96.4)$ & $28(100)$ & $28(100)$ & $28(100)$ \\
Itching & $10(35.7)$ & $14(50.0)$ & $11(39.3)$ & $16(57.1)$ \\
None & $18(64.3)$ & $14(50.0)$ & $17(60.7)$ & $12(42.9)$ \\
Mild & $10(35.7)$ & $13(46.4)$ & $10(35.7)$ & $14(50.0)$ \\
Moderate & - & $1(3.6)$ & $1(3.6)$ & $2(7.1)$ \\
Severe & - & - & - & - \\
Local heat & $5(17.9)$ & $1(3.6)$ & $5(17.9)$ & $4(14.3)$ \\
None & $23(82.1)$ & $27(96.4)$ & $23(82.1)$ & $24(85.7)$ \\
Mild & $5(17.9)$ & $1(3.6)$ & $5(17.9)$ & $4(14.3)$ \\
Moderate & - & - & - & - \\
Severe & - & - & - & - \\
\hline & & & & \\
\hline
\end{tabular}


Table 3 Demographic and Baseline Characteristics (Patients with PID)

\begin{tabular}{ll}
\hline Characteristic & MITT population $(N=38)$ \\
\hline Gender, $n(\%)$ & $21(55.3)$ \\
Female & $17(44.7)$ \\
Male & \\
Age (years) & $36.3(19.52)$ \\
Mean (SD) & $36.5(5-72)$ \\
Median (range) & \\
Age group, $n(\%)$ & $3(7.9)$ \\
5-11 years & $3(7.9)$ \\
12-15 years & $28(73.7)$ \\
16-64 years & $4(10.5)$ \\
65-72 years & \\
Race or ethnic group, $n(\%)$ & $37(97.4)$ \\
White & $1(2.6)$ \\
Black or African American & $2(5.3)$ \\
Hispanic or Latino & \\
Weight (kg) & $70.0(21.34)$ \\
Mean (SD) & $70.0(21-104)$ \\
Median (range) &
\end{tabular}

${ }^{\mathrm{a}}$ Ethnic groups were summarized independently of race.

${ }^{\mathrm{b}}$ Individual median values during the last 3 months of treatment with IVIG prior to this study.

necessity to have multiple injection sites, $n=7$ ), health issues present while receiving IVIG treatment and not resolved on SCIG $(n=4)$, feeling too good and not wanting to be reminded about the disease by weekly infusions ( $n=$ $2)$, and compliance issues $(n=1)$.

Patients' mean age was 36 years, including three children and three adolescents, and the proportion of males and females was similar (Table 3). Most of the patients had CVID; 32 patients had been diagnosed more than 2 years prior to the study. Two patients had XLA diagnosed more than 2 years prior to the study.

\section{Study Drug Administration}

All 38 MITT subjects received the intended 12 infusions during the wash-in/wash-out period. During the efficacy period, 23 subjects $(60.5 \%)$ received the planned 54 infusions, and 15 subjects (39.5\%) received between 11 and 53 infusions.

The mean IgPro20 dose during the wash-in/wash-out period ranged from 176.8 to $182.9 \mathrm{mg} / \mathrm{kg}$ bw per week. In the efficacy period, starting at week 13, the mean IgPro20 dose was 179.6 to $224.3 \mathrm{mg} / \mathrm{kg}$ bw per week.

Median infusion rate (total body) was $25 \mathrm{~mL} / \mathrm{h}$ during the wash-in/wash-out period (range 15.0-50.0 mL/h) and $50 \mathrm{~mL} / \mathrm{h}$ during the efficacy period (range $15.0-50.0 \mathrm{~mL} /$ h). Mean $( \pm S D)$ infusion rates (total body) were $30.9 \mathrm{~mL} / \mathrm{h}$ $( \pm 12.49)$ and $39.1 \mathrm{~mL} / \mathrm{h}( \pm 13.41)$, respectively. The overall median infusion duration was 2.0 hours (range 0.517.0 hours).

\section{Efficacy}

Primary Efficacy End Point No SBIs, as defined by the FDA, were observed in the MITT population in this study. The annual rate of SBIs per patient was 0 (upper $99 \%$ CI 0.132), and thus, the study objective was achieved (Table 4). There were no SBIs in the ITT population either.

Five AEs in three patients with CVID were examined closely by the review committee before identifying them as non-SBIs. In a 71-year-old woman, pneumonia was suspected twice during hospitalization for cellulitis and urinary tract infection (both serious AEs [SAEs]). In the first case, the diagnosis of pneumonia was rejected, as two x-rays and a computerized tomography (CT) chest scan did not reveal changes consistent with bacterial infection in lungs; repeat blood cultures were negative. The possibility of second pneumonia was raised because of nonproductive cough and focal areas of opacity in chest X-ray and CT scan. Pneumonia was rejected at the diagnosis because of the lack of new respiratory symptoms, normal white blood cell count, as well as negative bronchoalveolar lavage, Gram stain, and viral antigen panel results. In the same patient, an $\mathrm{AE}$ of suspected bacteremia during hospitalization for cellulitis was determined not to meet the criteria for SBI because an initial finding of Staphylococcus epidermidis, attributed to contaminated central venous catheter, was followed by multiple negative blood cultures and was not accompanied by the required diagnostic criteria for bacteremia/ septicemia. In a 60-year-old woman, an S. epidermidis infection during hospitalization for gastroenteritis (an SAE) was a suspected SBI of bacteremia, but because the single finding of infection associated with contaminated central venous catheter was followed by several sterile cultures and not accompanied by the required diagnostic criteria for bacteremia, the event was not considered an SBI. In a 17-year-old girl, an $\mathrm{AE}$ of pneumonia was not felt to meet SBI criteria. Although she was seen by her primary care physician for symptoms of cough and noted to have fever and crackles, none of the essential diagnostic features were present: productive cough, dyspnea or tachypnea, chills, chest pain, rigors, 
Table 4 Primary and Secondary Efficacy End Points (Patients with PID; MITT Population)
${ }^{\text {a }}$ Total number of days in the study.

${ }^{\mathrm{b}}$ Total number of days from patient diaries.

\begin{tabular}{lll}
\hline Efficacy end point & Number (\%) of patients & $\begin{array}{l}\text { Number (annual rate) } \\
\text { of events/days [CI] }\end{array}$ \\
\hline $\begin{array}{l}\text { Total no. of patients/study days } \\
\text { SBIs [upper 99\% CI] }\end{array}$ & $N=38$ & $N=12,697^{\mathrm{a}}$ \\
& $0(0)$ & $0(0)$ \\
Total no. of patients/study days & $N=38$ & $0.132]$ \\
Infection episodes (nonserious) [95\% CIs] & $31(81.6)$ & $N=12,697^{\mathrm{a}}$ \\
& & $96(2.76)$ \\
Total no. of patients/study days & $N=38$ & {$[2.235-3.370]$} \\
Days missed from work/school & $12(31.6)$ & $N=12,605^{\mathrm{b}}$ \\
& & $71(2.06)$ \\
Total no. of patients/study days & $N=38$ & $\mathrm{NA}$ \\
Days hospitalized due to infection & $1(2.6)$ & $N=12,605^{\mathrm{b}}$ \\
& & $7(0.2)$ \\
Total no. of patients/study days & $N=38$ & $\mathrm{NA}$ \\
Days with antibiotics for infection prophylaxis & $27(71.1)$ & $N=12,697^{\mathrm{a}}$ \\
or treatment & & $1,688(48.5)$ \\
\end{tabular}

headache, and sweats were absent; there was no dullness to percussion; white blood count was normal; hypoxemia was not found. Although a chest x-ray and blood or sputum cultures were not done, a throat swab sent for a rapid strep test was negative.

Secondary Efficacy End Points A total of 96 non-SBI infection episodes were observed in 31 (81.6\%) patients, resulting in an annual rate of 2.76 per patient $(95 \%$ CIs 2.235-3.370) (Table 4). Sinusitis was the most common infection experienced by 12 patients, followed by nasopharyngitis in 6 patients (14 and 11 patients in the ITT population, respectively).

Twelve patients (31.6\%) missed 71 days from work/ school (annual rate 2.06 per patient). A single patient was hospitalized for 7 days due to infections in the period between weeks 44 and 47 (annual rate 0.2 per patient). In total, 27 patients (71.1\%) spent 1,688 days on antibiotics (annual rate 48.5 days per patient) for treatment of AEs, prophylaxis, or medical/surgical/current conditions; 25 patients were treated for AEs during 1,040 days (annual rate 29.9 days per patient), and 2 patients had antibiotic prophylaxis for 16 days (annual rate 0.5 days per patient). Nine patients $(23.7 \%)$ used antibiotics on 664 days for the treatment of medical/surgical/current conditions, and one patient was treated with antibiotics for other indications (9 days).

In the MITT population, the mean $( \pm \mathrm{SD})$ of the individual median IgG trough levels was $12.56 \mathrm{~g} / \mathrm{L}( \pm 2.92 \mathrm{~g} / \mathrm{L})$ during the wash-in/wash-out period and $12.53 \mathrm{~g} / \mathrm{L}( \pm 3.21 \mathrm{~g} / \mathrm{L})$ during the efficacy period. Serum IgG levels stabilized by the end of the wash-in/wash-out period, when patients' individual doses were adjusted as described. Overall, mean serum IgG trough levels in the efficacy period were maintained between 12.1 and $12.9 \mathrm{~g} / \mathrm{L}$ (Fig. 1).

\section{Safety and Tolerability}

Local Reactions As expected, local reactions were observed in all 49 patients in the ITT population: 1,340 events were recorded during the 2,264 infusions, resulting in a rate

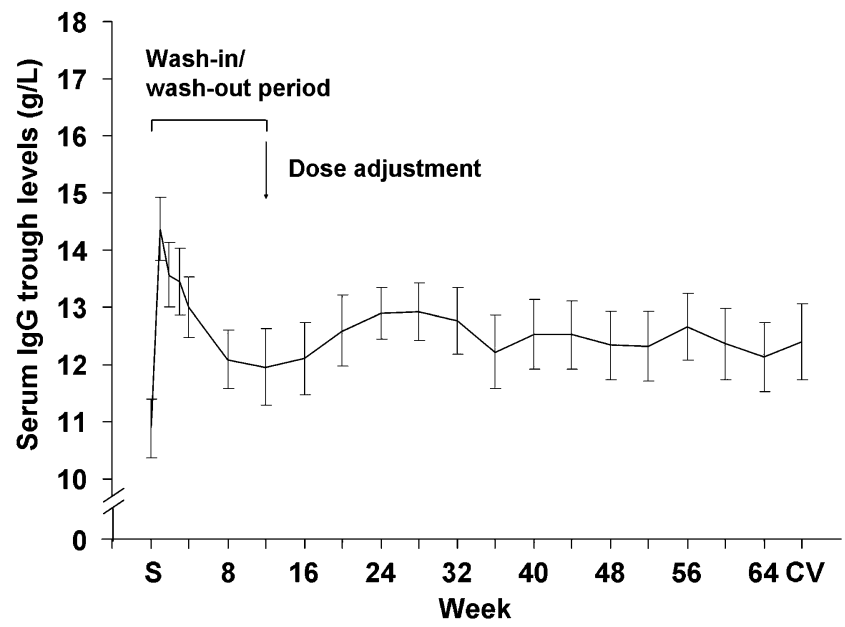

Fig. 1 Serum IgG trough levels (patients with PID). Blood samples were taken before infusion start at screening (S); at weeks 1, 2, 3, 4, 8, and 12; every 4 weeks thereafter; and at the completion visit (CV). For most infusions and for the completion visit, data from at least 29 patients were available; for the following infusions, the number of patients with available data was lower: infusion $60, n=28$; infusion 64 , $n=27$. Note that the patient's regular IVIG infusion was given just after screening, and SCIG administration was begun 1 week later 
Table 5 Local Reactions (Experienced by $\geq 2$ Patients $^{\mathrm{a}}$ with PID; ITT Population)

\begin{tabular}{lllll}
\hline AE & Mild & Moderate & Severe & Total \\
\hline Local reactions, $n$ (rate) $^{\mathrm{b}}$ & $1,251(0.553)$ & $85(0.038)$ & $4(0.002)$ & $1,340(0.592)$ \\
Injection site reaction, $n$ (rate) & $1,227(0.542)$ & $83(0.037)$ & $4(0.002)$ & $1,314(0.580)$ \\
Injection site bruising, $n$ (rate) & $19(0.008)$ & 0 & 0 & $19(0.008)$ \\
Injection site cyst, $n$ (rate) & $2(<0.001)$ & 0 & 0 & $2(<0.001)$ \\
\hline
\end{tabular}

$n$ number of events.

${ }^{a}$ Local reactions experienced by one patient each: injection site irritation, injection site nodule, injection site pain, all mild; infusion site scab and injection site eczema, both moderate

${ }^{\mathrm{b}}$ Rate was determined as number of events per infusion. The total number of infusions was 2,264.

of 0.592 events per infusion (Table 5). Of these, 1,322 events were temporally associated AEs (defined as occurring between the start of infusion and 72 hours after the end of infusion), and 1,338 events were considered at least possibly related to study medication. The most common AE was injection site reaction. Most local reactions were mild in intensity, and only four severe events were observed. Injection site reactions were predominantly mild (93.4\%); $6.3 \%$ were moderate and only $0.3 \%$ were severe. IgPro 20 dose had no effect on the incidence of local reactions (not shown). According to investigators' assessments 45 minutes after infusion, the overall incidence of injection site reactions remained stable over time (slope of linear regression line -0.0002), whereas according to patients' estimates 24 hours after infusion, it showed a slight tendency to decrease (slope of linear regression line -0.0018 ; Fig. 2). This apparent discrepancy may be due to the different times of assessment (immediate versus 24 hours postinfusion) or to the fact that patients were reporting less, as they learn to put up with the burdens of

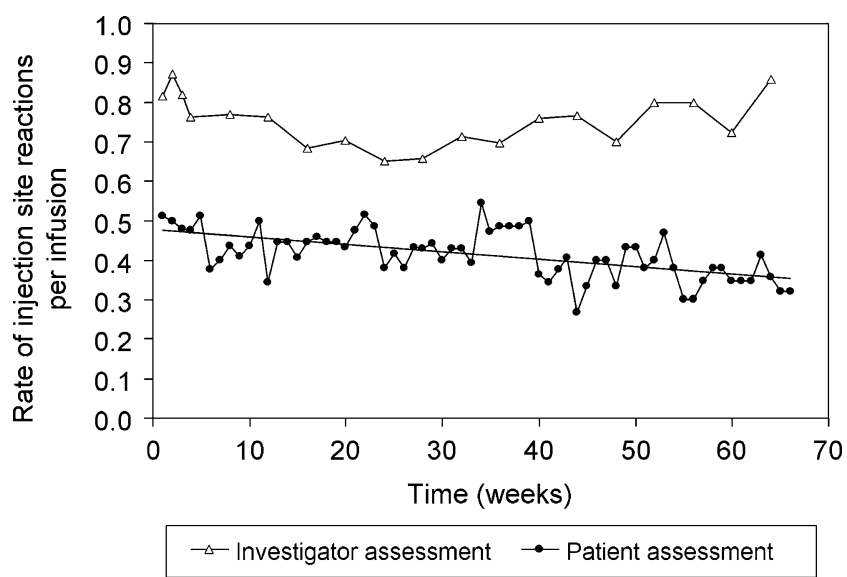

Fig. 2 Rate of injection site reactions over time (patients with PID). Investigator assessments were performed at 15 to 45 minutes after infusion at study visits (every 4 weeks). Patient assessments were made $24 \pm 3$ hours after every infusion until the completion visit (week 66). For both assessments, the number of infusions with available data decreased from 49 at week 1 to 28 at week 64 therapy. Patients tolerated the local reactions, and their assessment of tolerating these appeared to improve over time.

Overall AEs Excluding local reactions, 45 subjects (91.8\%) had at least one AE, of whom 25 patients $(51.0 \%)$ experienced AEs considered at least possibly related to study medication. Apart from local reactions following infusions, the most common $\mathrm{AE}$, regardless of relation to study treatment, was sinusitis, followed by headache and nasopharyngitis (Table 6). Most common related AEs are summarized in Table 7. Excluding local reactions, 409 AEs were reported in the 2,264 infusions administered in the study, resulting in a rate of 0.181 AEs per infusion. Of these, 98 AEs were considered at least possibly related to study medication (rate of 0.043 AEs per infusion).

Almost all AEs were of mild or moderate intensity (99\%). This was true for each of the three most common AEs as well: 8 of 20 AEs of sinusitis were mild and 12 were moderate; 21 of 40 AEs of headache were mild and 15 were moderate; 13 of 15 AEs of nasopharyngitis were mild and 2 were moderate. Nineteen severe AEs were reported, most occurring in one patient each. The most common severe AEs were headache (in four patients [8.2\%]), injection site reaction (in three patients [6.1\%]), and chest pain (in two patients [4.1\%]). Of the 19 severe AEs, 7 were considered related to study medication (4 infusion site reactions and 3 headaches).

Serious AEs There were no deaths during the study. Seven patients $(14.3 \%)$ experienced 10 SAEs, none of them an injection site reaction and none assessed as related to study medication. The SAEs were chest pain (2 events), gastroenteritis, obstruction of the small intestine, tooth abscess, cellulitis, urinary tract infection, decreased hemoglobin, musculoskeletal stiffness, and papillary thyroid cancer. Six of the SAEs were severe in intensity. Except for the SAE of papillary thyroid cancer, all SAEs resolved without sequelae by the final assessment. The SAE of cellulitis was of moderate intensity and was reported as an SAE because it 
Table 6 Most Common AEs (Experienced by $\geq 5$ Patients with PID; ITT Population)

\begin{tabular}{|c|c|c|c|c|}
\hline \multirow[t]{2}{*}{$\mathrm{AE}$} & \multicolumn{2}{|l|}{ All events } & \multicolumn{2}{|c|}{ Temporally associated $(72 \mathrm{~h})$} \\
\hline & $\begin{array}{l}\text { No. }(\%) \text { of } \\
\text { patients }(n=49)\end{array}$ & $\begin{array}{l}\text { No. (rate) of } \\
\text { events }(n=2,264)\end{array}$ & $\begin{array}{l}\text { No. }(\%) \text { of } \\
\text { patients }(n=49)\end{array}$ & $\begin{array}{l}\text { No. (rate) of } \\
\text { events }(n=2,264)\end{array}$ \\
\hline Any AE & $49(100)$ & $1,749(0.773)$ & $49(100)$ & $1,566(0.692)$ \\
\hline Injection site reaction & $49(100)$ & $1,314(0.580)$ & $49(100)$ & $1,298(0.573)$ \\
\hline Sinusitis & $14(28.6)$ & $20(0.009)$ & $7(14.3)$ & $10(0.004)$ \\
\hline Headache & $13(26.5)$ & $40(0.018)$ & $12(24.5)$ & $32(0.014)$ \\
\hline Nasopharyngitis & $11(22.4)$ & $15(0.007)$ & $8(16.3)$ & $8(0.004)$ \\
\hline Cough & $8(16.3)$ & $9(0.004)$ & $5(10.2)$ & $6(0.003)$ \\
\hline Diarrhea & $7(14.3)$ & $8(0.004)$ & $5(10.2)$ & $6(0.003)$ \\
\hline Bronchitis & $6(12.2)$ & $9(0.004)$ & $5(10.2)$ & $6(0.003)$ \\
\hline Fatigue & $6(12.2)$ & $6(0.003)$ & $4(8.2)$ & $4(0.002)$ \\
\hline Injection site bruising & $5(10.2)$ & $19(0.008)$ & $5(10.2)$ & $18(0.008)$ \\
\hline Back pain & $5(10.2)$ & $11(0.005)$ & $4(8.2)$ & $5(0.002)$ \\
\hline Acute sinusitis & $5(10.2)$ & $7(0.003)$ & $4(8.2)$ & $5(0.002)$ \\
\hline Nausea & $5(10.2)$ & $5(0.002)$ & $4(8.2)$ & $4(0.002)$ \\
\hline Abdominal pain upper & $5(10.2)$ & $5(0.002)$ & $3(6.1)$ & $3(0.001)$ \\
\hline Upper respiratory tract infection & $5(10.2)$ & $6(0.003)$ & $3(6.1)$ & $3(0.001)$ \\
\hline Rash & $5(10.2)$ & $7(0.003)$ & $2(4.1)$ & $3(0.001)$ \\
\hline
\end{tabular}

required hospitalization; it resolved without sequelae after 7 days. According to FDA criteria, cellulitis is not considered a potential SBI.

AEs Leading to Discontinuation Two patients (4.1\%) discontinued the study due to AEs. One patient, with a history of atopic dermatitis, asthma, and drug hypersensitivity, experienced a severe injection site reaction with lesions consistent with giant urticaria. The AE was considered at least possibly related to study medication; it resolved without sequelae after 13 days. Another patient experienced moderate myositis. Although this AE was

Table 7 Most Common Causally Related AEs (ITT Population)

\begin{tabular}{|c|c|c|c|c|}
\hline \multirow[b]{2}{*}{$\mathrm{AE}$} & \multicolumn{2}{|l|}{ Infusion-related } & \multicolumn{2}{|c|}{$\begin{array}{l}\text { Infusion-related and temporally } \\
\text { associated }(72 \mathrm{~h})\end{array}$} \\
\hline & $\begin{array}{l}\text { No. }(\%) \text { of } \\
\text { patients }(n=49)\end{array}$ & $\begin{array}{l}\text { No. (rate) of } \\
\text { events }(n=2,264)\end{array}$ & $\begin{array}{l}\text { No. }(\%) \text { of } \\
\text { patients }(n=49)\end{array}$ & $\begin{array}{l}\text { No. (rate) of } \\
\text { events }(n=2,264)\end{array}$ \\
\hline Any $\mathrm{AE}$ & $49(100)$ & $1,436(0.634)$ & $49(100)$ & $1,397(0.617)$ \\
\hline Injection site reaction & $49(100)$ & $1,313(0.580)$ & $49(100)$ & $1,297(0.573)$ \\
\hline Headache & $12(24.5)$ & $36(0.016)$ & $11(22.4)$ & $31(0.014)$ \\
\hline Injection site bruising & $5(10.2)$ & $19(0.008)$ & $5(10.2)$ & $18(0.008)$ \\
\hline Vomiting & $3(6.1)$ & $3(0.001)$ & $3(6.1)$ & $3(0.001)$ \\
\hline Pain & $3(6.1)$ & $4(0.002)$ & $2(4.1)$ & $3(0.001)$ \\
\hline Fatigue & $3(6.1)$ & $3(0.001)$ & $2(4.1)$ & $2(<0.001)$ \\
\hline Contusion & $2(4.1)$ & $3(0.001)$ & $2(4.1)$ & $3(0.001)$ \\
\hline Back pain & $2(4.1)$ & $3(0.001)$ & $2(4.1)$ & $2(<0.001)$ \\
\hline Diarrhea & $2(4.1)$ & $2(<0.001)$ & $2(4.1)$ & $2(<0.001)$ \\
\hline Abdominal pain upper & $2(4.1)$ & $2(<0.001)$ & $2(4.1)$ & $2(<0.001)$ \\
\hline Nausea & $2(4.1)$ & $2(<0.001)$ & $2(4.1)$ & $2(<0.001)$ \\
\hline Migraine & $2(4.1)$ & $3(0.001)$ & $1(2.0)$ & $2(<0.001)$ \\
\hline Rash & $2(4.1)$ & $2(<0.001)$ & $1(2.0)$ & $1(<0.001)$ \\
\hline Arthralgia & $2(4.1)$ & $2(<0.001)$ & $1(2.0)$ & $1(<0.001)$ \\
\hline
\end{tabular}


diagnosed posttreatment, it was recorded as the reason for discontinuation because the condition gradually worsened throughout the study. The patient had highly elevated alanine aminotransferase, aspartate aminotransferase, and creatine kinase at screening and a medical history that included leg pain. The AE was considered at least possibly related to study medication; it resolved several months after the final assessment.

Laboratory tests Median values and ranges of hematology and blood chemistry parameters showed no relevant changes over time. Most of the individual hematology and blood chemistry and all urinalysis values lying outside the normal range were considered by the investigator as not clinically significant.

No consistent or clinically relevant changes in vital signs or physical examination were observed. There was no clinical nor laboratory evidence of acquisition of any blood borne pathogen associated with study product.

Direct Coombs' test Forty-five patients had a negative direct Coombs' test at baseline, and five patients developed positive Coombs' tests at different visits. One of these five patients had low baseline hemoglobin (not considered clinically significant), with values normalizing during the study. The other four patients had hemoglobin within the normal range throughout the study, except one who had a value higher than the upper limit of the normal range at the completion visit (not considered clinically relevant).

No signs of hemolysis were observed in any patient. A decrease in hemoglobin of $\geq 2 \mathrm{~g} / \mathrm{dL}$ was recorded in five subjects but was not accompanied by a positive direct Coombs' result, a decrease in haptoglobin, or an increase in lactate dehydrogenase.

\section{Discussion}

The results of the Phase III study showed ideal efficacy of the first $20 \%$ SCIG IgPro20 in patients with PID. No SBIs were observed during the entire study period, and the rate of any infections was very low, 2.76 infections per patient per year. Mean serum IgG trough levels during the efficacy period were maintained between 12.1 and $12.9 \mathrm{~g} / \mathrm{L}$, which is within the normal range for healthy individuals. Local reactions were predominantly mild, and comprehensive safety monitoring showed no new types of AEs. Overall, the results of this study showed that IgPro20 is effective and well tolerated in this patient population. The development of a $20 \%$ IgG preparation with stable solution properties is a substantial technical achievement and represents a serious advance in patient convenience in $\operatorname{IgG}$ replacement therapy.
According to the 2008 FDA bulletin, patients with PID may experience four or more SBIs per year without immunoglobulin replacement therapy [26]. Children with XLA aged 2 to 111 months experienced 0.4 SBIs requiring hospitalization per patient per year before commencing immunoglobulin therapy [27]. SCIG products have been shown to be effective in preventing infections in patients with PID, with efficacy comparable to that of IVIG preparations [16]. The main efficacy end point in this study, the annual rate of SBIs, was 0 (upper 99\% CI 0.132) and thus much lower than the FDA requirement of 1 . Therefore, the primary efficacy objective of the study was met. This result compares well with the annual rate of 0.04 SBIs per patient obtained with Vivaglobin, the only SCIG product currently approved for use in the United States [19, 25]. Similar rates were reported also for three $10 \%$ liquid IVIG products. With Gammagard Liquid and Privigen, 0 and 0.08 SBIs per patient per year were observed, respectively [14, 28]. Despite the different definition of serious infections used in the study of Gamunex, a similar number was reported: $<0.2$ validated sinopulmonary infections per patient per year [29]. In general, the annual rate of SBIs in this study is slightly better than the overall mean of 0.068 SBIs per patient per year observed in the licensing trials of all IVIG preparations approved in the United States since 2000 [16].

The frequency of any infections observed in this study, 2.76 infections per patient per year, was lower than that reported in two Vivaglobin studies (4.43 infections per patient per year [25] and 4.1 respiratory per patient per year [19]) and lower than the combined mean value of 3.02 nonserious infections observed in the licensing trials of IVIG preparations approved in the United States since 2000 [16]. On average, patients missed 2.06 days per patient from work/school, which is lower than the annual rate of 3.70 days per patient reported for Vivaglobin [25].

IgPro20 showed very good tolerability with regard to both local injection site reactions and systemic AEs. Most (99\%) local reactions were mild or moderate, including injection site reactions, the most common $\mathrm{AE}, 99.7 \%$ of which were mild or moderate. Similar results were reported in a study of Vivaglobin performed in the Unites States: $91 \%$ of patients experienced infusion site reactions, and $96 \%$ of those were mild or moderate [25]. Direct comparison between IgPro20 and Vivaglobin in healthy volunteers showed that local tolerability of IgPro20 is similar to that of Vivaglobin, with a slight improvement in mean pain scores and intensity of erythema with IgPro20. Similar local tolerability results were observed with the use of IgPro16 and IgPro20, which may be attributed to the inherent qualities of the formulation. Tolerability of IgPro20 with regard to systemic AEs was also very good. 
In the phase III study described here, $24.5 \%$ of patients experienced temporally associated headache, a typical AE for immunoglobulin therapy, compared to $48 \%$ of patients treated with Vivaglobin for the same time period (64 weeks) [25]. Almost all headaches in this study were mild or moderate (36 of $40,90 \%$ ), compared to 20 of the 31 observed with Vivaglobin. Thus, it appears that both the local and systemic tolerability of IgPro20 was slightly better than that of Vivaglobin in both healthy subjects and patients with PID.

The safety profile of IgPro20 is indistinguishable from that of other immunoglobulin products, with nature, frequency, and relatedness of AEs typical of disease and treatment. Overall, the rate of infusion-related AEs other than local reactions was only 0.043 per infusion. No hemolysis was observed after IgPro20 administration. The frequency of typical, temporally associated AEs such as headache, fatigue, and nausea (Table 6) was less common with IgPro20 than with some recently tested IVIG preparations. Headache, fatigue, and nausea were reported by $36.1 \%, 16.4 \%$, and $9.8 \%$ of patients treated with Gammagard Liquid [28] and by $43.8 \%, 16.3 \%$, and $12.5 \%$ of patients treated with Privigen [14]. The remaining AEs observed with IgPro20 did not differ from AEs known to be typical for this therapy, and no new $\mathrm{AE}$ syndromes were observed. Immunologists treating adult patients with IVIG are often concerned over the potential risk of cardiac or thromboembolic AEs during the extreme IgG peak after infusion [30-32]. SCIG may provide additional safety in this respect.

Mean serum IgG levels achieved in this study were within the normal range for healthy individuals and higher than the recommended minimal trough level of $5 \mathrm{~g} / \mathrm{L}$ [33]. In this U.S. study, systemic exposure equivalent to previous IVIG treatment was targeted, and therefore, a $153 \%$ dose adjustment (to achieve equivalent bioavailability with SCIG) was performed after the wash-in/wash-out period (week 12). In a recently completed European study of IgPro20 in patients with PID, subcutaneous therapy was started and maintained at weekly doses equivalent to one fourth of the previous monthly IVIG doses to achieve comparable serum IgG trough levels, as recommended by the European Medicines Agency [34]. It remains to be established whether one of these strategies proves better for achieving optimal clinical outcomes.

IgPro20 was largely self-administered by patients at home. The relatively low infusion volumes of subcutaneous infusions resulted in a median infusion duration of only 2 hours allowing for convenient treatment with the possibility of improved patient acceptability and possibly improved compliance. Some patients withdrew consent (14 patients) due to personal reasons, suggesting that certain patients may prefer IVIG over SCIG. It has been reported previously that some patients prefer intravenous to subcutaneous $\operatorname{IgG}$ administration, primarily because they are uncomfortable with self-infusion or fear facing AEs at home [35]. Thus, the decision regarding the most appropriate modality of immunoglobulin replacement should continue to be individualized with an understanding of patient-specific preferences as well as risks and goals of treatment.

In conclusion, IgPro20 is the first $20 \%$ SCIG and shows excellent efficacy and tolerability in patients with PID. IgPro20 should provide an important alternative for patients who would like to benefit from convenient selfadministration of immunoglobulin therapy at home.

Acknowledgements This study was supported by CSL Behring LLC, King of Prussia, PA. The opinions expressed in this paper are those of the authors.

The authors thank Dr. Christian Peters for his help during the conduct of the study, and Dr. Andrea Sebald and Dr. Martin Bexon for critical review of the manuscript. The assistance of Fritz Schindel, Corina Miede, and Cordula Massion from Accovion $\mathrm{GmbH}$ for the statistical analyses is gratefully appreciated. We also acknowledge the editorial assistance of Phocus Services Ltd supported by CSL Behring.

Open Access This article is distributed under the terms of the Creative Commons Attribution Noncommercial License which permits any noncommercial use, distribution, and reproduction in any medium, provided the original author(s) and source are credited.

\section{References}

1. Primary immunodeficiency diseases: report of an IUIS Scientific Committee. Clin Exp Immunol. 1999;118:1-28.

2. Buckley RH. Primary immunodeficiency diseases due to defects in lymphocytes. N Engl J Med. 2000;343:1313-24.

3. Chapel H, Geha R, Rosen F. Primary immunodeficiency diseases: an update. Clin Exp Immunol. 2003;132:9-15.

4. Cunningham-Rundles C, Bodian C. Common variable immunodeficiency: clinical and immunological features of 248 patients. Clin Immunol. 1999;92:34-48.

5. Ochs HD, Smith CIE, Puck JM. Primary immunodeficiency diseases: a molecular and genetic approach. Secondth ed. New York: Oxford University Press Inc, USA; 2007.

6. Park MA, Li JT, Hagan JB, Maddox DE, Abraham RS. Common variable immunodeficiency: a new look at an old disease. Lancet. 2008;372:489-502.

7. Berger M. Principles of and advances in immunoglobulin replacement therapy for primary immunodeficiency. Immunol Allergy Clin North Am. 2008;28:413-37.

8. Busse PJ, Razvi S, Cunningham-Rundles C. Efficacy of intravenous immunoglobulin in the prevention of pneumonia in patients with common variable immunodeficiency. J Allergy Clin Immunol. 2002;109:1001-4.

9. Bussel JB, Eldor A, Kelton JG, Varon D, Brenner B, Gillis S, et al. IGIV-C, a novel intravenous immunoglobulin: evaluation of safety, efficacy, mechanisms of action, and impact on quality of life. Thromb Haemost. 2004;91:771-8.

10. Church JA, Borte M, Taki H, Nelson RP, Sleasman JW, Knutsen $\mathrm{AP}$, et al. Efficacy and safety of Privigen in children and adolescents with primary immunodeficiency. Pediatr Asthma Allergy Immunol. 2009;22:53-62. 
11. de Gracia J, Vendrell M, Alvarez A, Pallisa E, Rodrigo MJ, de la $\mathrm{RD}$, et al. Immunoglobulin therapy to control lung damage in patients with common variable immunodeficiency Int Immunopharmacol. 2004;4:745-53.

12. Eijikhout HW, van der Meer JW, Kallenberg CG, Weening RS, van Dissel JT, Sanders LA, et al. The effect of two different dosages of intravenous immunoglobulin on the incidence of recurrent infections in patients with primary hypogammaglobulinemia. A randomized, double-blind, multicenter crossover trial. Ann Intern Med. 2001;135:165-74.

13. Gardulf A, Hammarstrom L, Smith CI. Home treatment of hypogammaglobulinaemia with subcutaneous gammaglobulin by rapid infusion. Lancet. 1991;338:162-6.

14. Stein MR, Nelson RP, Church JA, Wasserman RL, Borte M, Vermylen C, et al. Safety and efficacy of Privigen ${ }^{\circledR}$, a novel $10 \%$ liquid immunoglobulin preparation for intravenous use, in patients with primary immunodeficiencies. J Clin Immunol. 2009;29:137-44.

15. Abrahamsen TG, Sandersen H, Bustnes A. Home therapy with subcutaneous immunoglobulin infusions in children with congenital immunodeficiencies. Pediatrics. 1996;98:1127-31.

16. Berger M. Subcutaneous administration of IgG. Immunol Allergy Clin North Am. 2008;28:779-802. viii.

17. Chapel HM, Spickett GP, Ericson D, Engl W, Eibl MM, Bjorkander J. The comparison of the efficacy and safety of intravenous versus subcutaneous immunoglobulin replacement therapy. J Clin Immunol. 2000;20:94-100.

18. Gardulf A, Andersen V, Bjorkander J, Ericson D, Froland SS, Gustafson R, et al. Subcutaneous immunoglobulin replacement in patients with primary antibody deficiencies: safety and costs. Lancet. 1995;345:365-9.

19. Gardulf A, Nicolay U, Asensio O, Bernatowska E, Bock A, Carvalho BC, et al. Rapid subcutaneous IgG replacement therapy is effective and safe in children and adults with primary immunodeficiencies - a prospective, multi-national study. J Clin Immunol. 2006;26:177-85.

20. Gardulf A, Nicolay U. Replacement IgG therapy and self-therapy at home improve the health-related quality of life in patients with primary antibody deficiencies. Curr Opin Allergy Clin Immunol. 2006;6:434-42.

21. Hansen S, Gustafson R, Smith CI, Gardulf A. Express subcutaneous IgG infusions: decreased time of delivery with maintained safety. Clin Immunol. 2002;104:237-41.

22. Stiehm ER, Casillas AM, Finkelstein JZ, Gallagher KT, Groncy PM, Kobayashi RH, et al. Slow subcutaneous human intravenous immunoglobulin in the treatment of antibody immunodeficiency: use of an old method with a new product. J Allergy Clin Immunol. 1998;101:848-9.
23. Welch MJ, Stiehm ER. Slow subcutaneous immunoglobulin therapy in a patient with reactions to intramuscular immunoglobulin. J Clin Immunol. 1983;3:285-6.

24. Stucki M, Boschetti N, Schaefer W, Hostettler T, Kaesermann F, Nowak T, et al. Investigations of prion and virus safety of a new liquid IVIG product. Biologicals. 2008;36:239-47.

25. Ochs HD, Gupta S, Kiessling P, Nicolay U, Berger M. Safety and efficacy of self-administered subcutaneous immunoglobulin in patients with primary immunodeficiency diseases. J Clin Immunol. 2006;26:265-73.

26. FDA. Safety, efficacy, and pharmacokinetic studies to support marketing of immune globulin intravenous (human) as replacement therapy for primary humoral immunodeficiency. Guidance for industry; 2008.

27. Quartier P, Debre M, De Blic J, de Sauverzac R, Sayegh N, Jabado N, et al. Early and prolonged intravenous immunoglobulin replacement therapy in childhood agammaglobulinemia: a retrospective survey of 31 patients. J Pediatr. 1999;134:589-96.

28. Church JA, Leibl H, Stein MR, Melamed IR, Rubinstein A, Schneider LC, et al. Efficacy, safety and tolerability of a new $10 \%$ liquid intravenous immune globulin [IGIV 10\%] in patients with primary immunodeficiency. J Clin Immunol. 2006;26:388-95.

29. Roifman CM, Schroeder H, Berger M, Sorensen R, Ballow M, Buckley RH, et al. Comparison of the efficacy of IGIV-C, $10 \%$ (caprylate/chromatography) and IGIV-SD, $10 \%$ as replacement therapy in primary immune deficiency. A randomized doubleblind trial Int Immunopharmacol. 2003;3:1325-33.

30. Dave S, Hagan J. Myocardial infarction during intravenous immunoglobulin infusion in a 65 -year-old man with common variable immunodeficiency and subsequent successful repeated administration. Ann Allergy Asthma Immunol. 2007;99:567-70.

31. Caress JB, Hobson-Webb L, Passmore LV, Finkbiner AP, Cartwright MS. Case-control study of thromboembolic events associated with IV immunoglobulin. J Neurol. 2009;256:339-42.

32. Brown HC, Ballas ZK. Acute thromboembolic events associated with intravenous immunoglobulin infusion in antibody-deficient patients. J Allergy Clin Immunol. 2003;112:797-9.

33. Stiehm ER. Human intravenous immunoglobulin in primary and secondary antibody deficiencies. Pediatr Infect Dis J. 1997;16:696-707.

34. European Agency for the Evaluation of Medicinal Products (EMEA), Committee for Proprietary Medicinal Products (CPMP). Note for guidance on the clinical investigation of human normal immunoglobulin for subcutaneous and intramuscular use. EMEA/ CPMP/BPWG/283/00; 2002

35. Kittner JM, Grimbacher B, Wulff W, Jager B, Schmidt RE. Patients' attitude to subcutaneous immunoglobulin substitution as home therapy. J Clin Immunol. 2006;26:400-5. 